\title{
Comparação dos fatores de risco para amputações maiores e menores em pacientes diabéticos de um Programa de Saúde da Família
}

\author{
Comparison of risk factors for major and minor amputation in diabetic \\ patients included in a Family Health Program
}

\author{
Elvira Cancio Assumpção ${ }^{1}$, Guilherme Benjamin Pitta ${ }^{2}$, Ana Carolina Lisboa de Macedo ${ }^{1}$, Gustavo \\ Borges de Mendonça ${ }^{1}$, Larissa Christyne Araújo de Albuquerque ${ }^{1}$, Lívia Cavalcanti Braga de Lyra ${ }^{1}$, \\ Raquel Menezes Timbó ${ }^{1}$, Ticiana Leal Leite Buarque ${ }^{3}$
}

\section{Resumo}

Contexto: Dentre as maiores causas de internamento hospitalar em pacientes com diabetes melito tipos 1 e 2 estão as complicações do pé diabético, principalmente pelas sequelas, muitas vezes incapacitantes, destacando-se as amputações de membros inferiores. A insuficiência vascular periférica ocorre mais precocemente nesses pacientes. A coexistência de neuropatia, isquemia e imunodeficiência favorece o desenvolvimento de infecções nos membros inferiores, que, se não tratadas adequadamente, podem levar a amputações e até à morte.

Objetivos: Comparar os fatores de risco para amputações maiores e menores em pacientes diabéticos de um Programa de Saúde da Família do CAIC Virgem dos Pobres III, em Maceió, AL.

Métodos: Foram examinados 93 pacientes com o diagnóstico de diabetes melito, sendo avaliada a realização ou não de amputações de membros inferiores. As variáveis analisadas foram: sexo, idade, tipo do diabetes, pressão arterial, amputação prévia (se maior ou menor), alterações dermatológicas, alterações de pulsos arteriais pedioso e tibial posterior, deformidades e neuropatia, e foram classificadas de acordo com a classificação de Wagner e de Texas.

Resultados: Todos os pacientes eram diabéticos tipo 2. Verificou-se que $4,30 \%$ dos pacientes evoluíram para amputação de membros inferiores. Não se observou variação significativa da hipertensão, deformidades e neuropatia em relação ao grupo de pacientes que foram amputados. Entretanto, a ausência de detecção dos pulsos distais dos membros inferiores revelou-se bastante significativa com relação ao desfecho de amputação.

Conclusão: Deve-se proporcionar aos diabéticos um atendimento ambulatorial adequado para que seja possível prevenir ou minimizar tais complicações.

Palavras-chave: Amputação, pé diabético, diabetes melito.

\begin{abstract}
Background: The main causes of hospital admission in patients with type 1 and 2 diabetes mellitus include diabetic foot complications, which may result in particularly disabling sequelae, such as lower limb amputation. Peripheral vascular insufficiency is a common early occurrence in these patients. The coexistence of neuropathy, ischemia, and immunodeficiency favors the development of infections in the lower limbs, which if not treated properly can lead to amputation and even death.
\end{abstract}

Objective: Compare risk factors for major and minor amputations in diabetic patients in the Family Health Program of the health care facility CAIC Virgem dos Pobres III, in Maceió, state of Alagoas, Brazil.

Methods: We examined 93 patients diagnosed with diabetes, assessing whether or not lower limb amputation was performed. The variables analyzed were: sex, age, type of diabetes, blood pressure, previous amputation (whether major or minor), skin changes, changes in arterial pedal and posterior tibial pulses, deformities, and neuropathy. Variables were classified according to the Wagner and Texas wound classification.

Results: All patients were diagnosed with type 2 diabetes. We found that $4.30 \%$ of the patients progressed to lower limb amputation. There was no significant variation in hypertension, deformities and neuropathy in relation to the amputee group. However, absence of distal pulses in the lower limb proved to be quite significant in relation to amputation outcome.

Conclusion: Diabetic patients should receive appropriate outpatient medical care in order to prevent or minimize diabetes-related complications.

Keywords: Amputation, diabetic foot, diabetes mellitus.

\footnotetext{
1. Acadêmicos de Medicina, Universidade de Ciências da Saúde de Alagoas (UNCISAL), Maceió, AL.

2. Doutor. Professor adjunto, Departamento de Cirurgia, UNCISAL, Maceió, AL.

3. Fisioterapeuta, UNCISAL, Maceió, AL. Mestranda, Reabilitação Pulmonar, Universidade Federal de São Paulo (UNIFESP), São Paulo, SP. Especialização, Fisioterapia Cardiopulmonar, UNCISAL, Maceió, AL.
}

Trabalho de conclusão de graduação médica financiado pela Liga Acadêmica Vascular Prof. Dr. Emil Burihan (LAVA).

Não foram declarados conflitos de interesse associados à publicação deste artigo.

Artigo submetido em 16.09.08, aceito em 05.05.09.

J Vasc Bras. 2009;8(2):133-138.

Copyright $\odot 2009$ by Sociedade Brasileira de Angiologia e de Cirurgia Vascular 


\section{Introdução}

Os doentes diabéticos têm um risco 15 vezes maior de serem submetidos a amputações, sendo mais frequentes na população diabética de baixo nível socioeconômico, com condições inadequadas de higiene e pouco acesso aos serviços de saúde ${ }^{1-3}$. Quando os pacientes procuram atendimento médico, as lesões geralmente estão em estágios avançados, requerendo tratamento cirúrgico, que muitas vezes os incapacitam para suas atividades de rotina ${ }^{4,5}$. O pé diabético causa considerável sofrimento, mudanças no estilo e qualidade de vida do paciente, impedindo suas funções normais e, finalmente, em alguns casos, levando à amputação, com consequências extremas, como, por exemplo, sobrecarga do sistema previdenciário por aposentadorias precoces, perda das funções laborais em faixa etária produtiva e altos custos hospitalares para tratamento e reabilitação ${ }^{1,6,7}$. A insuficiência vascular periférica ocorre mais precocemente nos diabéticos que nos não-diabéticos, e as artérias do segmento infrapatelar são acometidas com maior frequência. A isquemia pode contribuir ou ser considerada causa para progressão de lesões nos pés, que agem de forma favorável para o desenvolvimento de infecções. A coexistência de neuropatia, isquemia e imunodeficiência favorecem o desenvolvimento de infecções extensas e severas em membros inferiores, que, se não tratadas adequadamente, podem levar a amputações e até ao óbito ${ }^{1}$. Estudos indicam que as ulcerações nos pés diabéticos precedem cerca de $85 \%$ das amputações de extremidades inferiores. A longa duração da doença, hiperglicemia prolongada, dislipidemia, hábitos de fumar e ingerir bebida alcoólica e presença de neuropatia, de doença vascular periférica e de lesões ulcerativas prévias são alguns dos fatores de risco para amputações em pessoas com diabetes melito $^{8-11}$. As amputações definidas como "maiores" são aquelas em que ocorre amputação da parte proximal do pé, perna (abaixo do joelho), coxa (acima do joelho) e desarticulação do quadril; enquanto que as "menores" são restritas aos dedos dos pés ou à parte anterior do pé, sendo assim, de certa forma, mais bem aceitas pelos pacientes porque dispensam o uso de próteses e não impossibilitam a deambulação ${ }^{1,10}$. Para que seja possível melhorar os cuidados com o paciente diabético é essencial combinar a profilaxia para diabetes melito e o controle e o tratamento das lesões, conjuntamente com o tratamento cirúrgico, antimicrobiano e da doença vascular periférica, visando diminuir a morbimortalidade relacionada ao diabetes melito ${ }^{1,11}$.

\section{Métodos}

A amostra totalizava 130 pacientes já com o diagnóstico estabelecido de diabetes melito tipo 1 ou 2, cadastrados no Programa de Saúde da Família (PSF) Virgem dos Pobres III, localizado na Avenida Senador Rui Palmeira, sem $\mathrm{n}^{\mathrm{o}}$, Trapiche da Barra, Maceió (AL). Foram excluídos da pesquisa os pacientes que faleceram, mulheres grávidas, índios e aqueles que apresentaram algum prejuízo à sua autonomia, como distúrbios psiquiátricos. A pesquisa foi submetida à avaliação e aprovação do Comitê de Ética em Pesquisa da Universidade de Ciências da Saúde de Alagoas (protocolo $n^{\circ}$ 895) e foi realizada de acordo com a Resolução 196/96 do Conselho Nacional de Saúde e com a Declaração de Helsinki. A coleta da amostra foi realizada de forma consecutiva com os pacientes diabéticos cadastrados no PSF, na unidade Virgem dos Pobres III, de acordo com a disposição de distribuição dos agentes de saúde. Baseado no protocolo pré-estabelecido, 93 pacientes diabéticos foram avaliados no período de junho-julho de 2008 e, após consentimento, submetidos a exame clínico, com anamnese e exame físico. Considerou-se como variável primária a realização ou não de amputações de membros inferiores. As variáveis analisadas em relação a essa foram: sexo, idade, tipo do diabetes, pressão arterial $\geq 140 \mathrm{x}$ $90 \mathrm{mmHg}^{12}$, ocorrência de amputação prévia, presença de alterações dermatológicas - como ressecamento, fissuras, palidez, hiperemia, úlcera ativa, onicomicose -, alterações de pulsos arteriais pedioso e tibial posterior, presença de deformidades e de neuropatia (foi utilizado monofilamento de Semmes-Weinstein $10 \mathrm{~g}$ para determinar a percepção de pressão em ambos os pés, em 10 locais diferentes). Os pacientes foram também avaliados em relação ao nível de amputação, se eram maiores ou menores. Não foi realizada distinção entre os pés diabéticos entre neuropáticos, infecciosos, isquêmicos ou mistos. Dentre os pacientes diabéticos tipos 1 e 2, as lesões apresentadas por eles foram enquadradas de acordo com a classificação de Wagner:

- Grau 0: pé em risco, presença de fissura interdigital ou no calcâneo, sem infecção aparente;

- Grau 1: infecção superficial micótica e/ou bacteriana leves;

- Grau 2: infecção profunda, atingindo tecido celular subcutâneo, tendões e ligamentos, sem osteomielite; 
- Grau 3: infecção profunda, com abscesso na região média do pé, com tendinite ou sinovite purulentas e osteomielite;

- Grau 4: infecção e gangrena localizada em dedos, região plantar anterior e calcanhar;

- Grau 5: infecção e gangrena de todo o pé.

Foi utilizada também a classificação da Universidade do Texas para avaliação e classificação do pé diabético:

- 0: ausência de patologia (ausência de história de ulceração e sensibilidade protetora intacta);

- 1: neuropatia sem deformidade (ausência de deformidade do pé e de sensibilidade protetora);

- 2: neuropatia com deformidade (presença de deformidade do pé e ausência de sensibilidade protetora;

- 3: história de patologia (história de articulação de Charcot e/ou ulceração neuropática, presença de deformidade do pé);

- 4A: pé com ulceração neuropática (presença de ulceração neuropática não-infectada);

- 4B: pé com neuro-artropatia de Charcot aguda (presença de articulação de Charcot);

- 5: pé diabético infectado (presença de ferida infectada);

- 6: pé diabético vascular (comprometimento da perfusão sanguínea).

Com vistas a avaliar a interdependência entre as variáveis citadas, utilizou-se o teste de Fisher. A decisão estatística foi feita com base no valor descritivo do teste (valor de p). Na análise multivariada utilizou-se o odds ratio (OR) como medida de associação.

\section{Resultados}

Dentre os 130 pacientes diabéticos que estavam cadastrados no PSF do CAIC Virgem dos Pobres III, 37 não foram examinados, cinco não residiam mais no local, oito haviam falecido e 24 não se encontravam em sua residência no momento das três visitas realizadas. Dos 93 pacientes examinados, $32,3 \%$ eram do sexo masculino e $67,7 \%$ do sexo feminino. A idade dos pacientes estava entre $30 \mathrm{e}$ 82 anos, sendo a média 58,5 anos, com desvio padrão de 11,49 . Todos os pacientes eram diabéticos tipo 2. Dos pacientes avaliados, 4,30\% (4/93) evoluíram para algum tipo de amputação de membros inferiores, sendo que desses
$75 \%$ (3/4) eram do sexo feminino, 25\% (1/4) do sexo masculino e 3,2\% (3/93) apresentaram história de úlcera prévia. A média de idade dos pacientes submetidos à amputação foi de 61,75 anos. O relato de amputação prévia foi verificado em 50\% (2/4). Do total de amputações prévias, $50 \%$ eram amputações maiores e 50\% amputações menores. Dentre os 93 pacientes, 62,4\% (58/93) estavam normotensos, enquanto que 37,6\% (35/93) apresentaram elevação da pressão arterial no momento da avaliação. $\mathrm{Na}$ avaliação dermatológica dos pés, apenas 15 pacientes, ou seja, 16,1\% (15/93), não apresentavam alterações dermatológicas, enquanto que $83,9 \%$ (78/93) tinham algum tipo de alteração, como ressecamento (68,8\%), fissuras $(37,63 \%)$ e palidez em $18,27 \%$ dos pacientes. Alguns deles apresentavam associação de mais de uma alteração dermatológica. $\mathrm{Na}$ avaliação neurológica realizada meio do filamento Semmes-Weinstein $10 \mathrm{~g}$, descobriu-se que 49,5\% (46/93) dos pacientes não apresentavam alteração na sensibilidade e que 50,5\% (47/93) tinham a sensibilidade alterada, sugerindo neuropatia diabética. Ao exame físico, observou-se que $94,6 \%$ não possuíam deformidade nos pés e $5,4 \%$ possuíam algum tipo de deformidade. A avaliação com o teste de Fisher não detectou significância para as variáveis amputação versus hipertensão $(\mathrm{p}=0,630)$, presença de deformidades ( $p=0,201)$, avaliação neurológica $(p=0,117)$, classificação de Wagner $(p=0,064)$ e amputação prévia $(\mathrm{p}=0,125)$, aceitando-se, portanto, a hipótese de independência entre cada uma dessas variáveis e o desfecho de amputação do membro inferior (Tabela 1).

Em relação a amputação versus pulsos arteriais, o teste de Fisher demonstrou que existe diferença significativa entre tais variáveis. Verificou-se que a ocorrência de amputação foi significativamente mais frequente que quando relacionada à ausência de pulsos $(\mathrm{p}<0,05)$. Só não ficou evidente quando se relacionou o pulso tibial posterior esquerdo (Tabela 2). Enquanto se analisa a gravidade das lesões através da classificação de Wagner, verificou-se que $96,8 \%(90 / 93)$ dos doentes apresentaram grau igual a 0 , enquanto 2,2\% (2/93) apresentaram grau 1. Desses, 1,1\% (1/93) foram classificados como grau 2.

Não foi possível aplicar nenhum teste estatístico na classificação de Texas, mas pode-se verificar a prevalência dos pacientes em cada tipo de acordo com tal classificação (Tabela 3). 
Tabela 1 - Hipertensão, deformidades, avaliação neurológica, classificação de Wagner e úlcera prévia entre os pacientes com e sem amputação

\begin{tabular}{|c|c|c|c|c|}
\hline \multirow[t]{2}{*}{ Variável } & \multicolumn{2}{|c|}{ Amputação } & \multirow[t]{2}{*}{ Total } & \multirow[t]{2}{*}{$\mathrm{p}$} \\
\hline & Não & Sim & & \\
\hline \multicolumn{5}{|c|}{ Hipertensão } \\
\hline Não & 56 & 2 & 58 & \\
\hline Sim & 33 & 2 & 35 & \\
\hline Total & 89 & 4 & 93 & 0,630 \\
\hline \multicolumn{5}{|c|}{ Deformidades } \\
\hline Não & 85 & 3 & 88 & \\
\hline Sim & 4 & 1 & 5 & \\
\hline Total & 89 & 4 & 93 & 0,201 \\
\hline \multicolumn{5}{|c|}{ Avaliação neurológica } \\
\hline Alterada & 43 & 4 & 47 & \\
\hline Normal & 46 & 0 & 46 & \\
\hline Total & 89 & 4 & 93 & 0,117 \\
\hline \multicolumn{5}{|c|}{ Classificação de Wagner } \\
\hline 0 & 88 & 2 & 90 & \\
\hline 1 & 1 & 1 & 2 & \\
\hline Total & 89 & 3 & 92 & 0,064 \\
\hline \multicolumn{5}{|c|}{ Úlcera prévia } \\
\hline Não & 87 & 3 & 90 & \\
\hline Sim & 2 & 1 & 3 & \\
\hline Total & 89 & 4 & 93 & 0,125 \\
\hline
\end{tabular}

\section{Discussão}

Sabe-se que as complicações do pé diabético são frequentes e responsáveis por cerca de $20 \%$ de hospitalizações dos pacientes diabéticos ${ }^{7}$. Sabe-se, ainda, que tais pacientes têm um risco 15 vezes maior de serem submetidos a amputações, sendo mais frequentes na população diabética de baixo nível socioeconômico, com condições inade-
Tabela 3 - Classificação de Texas

\begin{tabular}{lccc}
\hline Escore & Frequência & Porcentagem & Porcentagem válida \\
\hline 0 & 48 & 51,6 & 51,6 \\
1 & 32 & 34,4 & 34,4 \\
2 & 7 & 7,5 & 7,5 \\
3 & 2 & 2,2 & 2,2 \\
$4 \mathrm{~A}$ & 1 & 1,1 & 1,1 \\
$4 \mathrm{~B}$ & 0 & 0 & 0 \\
5 & 1 & 1,1 & 1,1 \\
6 & 2 & 2,2 & 2,2 \\
Total & 93 & 100 & 100 \\
\hline
\end{tabular}

quadas de higiene e pouco acesso aos serviços de saú$\mathrm{de}^{4,5,13}$. A intenção deste estudo é detectar os fatores de risco envolvidos nas amputações maiores e menores, bem como a ocorrência dessas numa comunidade de baixa renda assistida pelo PSF. Sabendo da importância das ações no que se refere à prevenção e ao tratamento das complicações do pé diabético, foi desenvolvido um estudo transversal e descritivo para avaliar a associação entre amputações em pacientes diabéticos e fatores de risco para essa ocorrência. Tal estudo visa à redução das taxas de amputações através de uma melhor orientação para o planejamento de ações terapêuticas e de profilaxia, e, consequentemente, tentar minimizar a realização de procedimentos que poderiam ser, ao menos em parte, evitados ${ }^{5,14}$. A amostra da população estudada é de pacientes que recebem tratamento ambulatorial, podendo, então, ter ocorrido um viés de seleção. A seleção do trabalho ocorreu em decorrência da escassez de estudos que avaliem a ocorrência de amputações em pacientes diabéticos, correlacionando-as a possíveis fatores de riscos em uma comunidade de baixa renda acompanhada pelo PSF. Durante os meses de junho e julho, 93 pacientes foram avaliados, e observou-se que todos eram diabéticos tipo 2, não sendo possível avaliar as variá-

Tabela 2 - Relação entre pulsos arteriais pedioso e tibial posterior

\begin{tabular}{|c|c|c|c|c|c|c|c|c|}
\hline \multirow[t]{2}{*}{ Amputação } & \multicolumn{8}{|c|}{ Pulsos } \\
\hline & PD presente & PD ausente & PE presente & PE ausente & TPD presente & TPD ausente & TPE presente & TPE ausente \\
\hline Não & 69 & 5 & 64 & 8 & 45 & 6 & 49 & 9 \\
\hline Sim & 1 & 2 & 1 & 2 & 0 & 3 & 1 & 2 \\
\hline $\mathrm{p}$ & & 0,021 & & 0,045 & & 0,003 & & 0,081 \\
\hline
\end{tabular}

$\mathrm{PD}=$ pedioso direito; $\mathrm{PE}=$ pedioso esquerdo $\mathrm{TPD}=$ tibial posterior direito; $\mathrm{TPE}=$ tibial posterior esquerdo. 
veis propostas nos diabéticos tipo 1. A maioria era do sexo feminino (67,7\%). De acordo com estudos de Moss et al. ${ }^{1,15}$, o sexo masculino é um fator de risco para amputações em diabéticos tipo 2 , mas do total avaliado no presente estudo, 4,3\% evoluíram para algum tipo de amputação de membros inferiores, sendo que, desses, 75\% (3/4) foram do sexo feminino e $25 \%$ (1/4) do sexo masculino, não havendo, portanto, maior prevalência dos homens. A média de idade dos pacientes que evoluíram para amputação foi de 61,75 anos, evidenciando que estão mais susceptíveis a partir da sexta década de vida ${ }^{5}$. As complicações crônicas, como neuropatia, parecem independer do sexo, sendo mais relacionadas ao controle glicêmico, embora já tenha sido demonstrada maior mortalidade no sexo masculino em decorrência de infecções ${ }^{16}$. De acordo com Gamba et al. ${ }^{9}$, a hipertensão arterial contribui para o desenvolvimento e progressão das complicações crônicas do diabetes melito, embora não tenham sido observadas diferenças estatisticamente significativas entre amputações e hipertensão arterial $(\mathrm{p}=0,630)$ e amputações e deformidade ( $p=0,201)$, de acordo com o teste de Fisher neste estudo. A análise através do odds ratio também não evidenciou tal relação. Uma possível explicação para isso é que, na presença das demais variáveis, a associação entre amputações e hipertensão perdem sua intensidade. Contudo, segundo a Sociedade Brasileira de Diabetes ${ }^{17}$, a hipertensão se comporta como um fator de risco, quando comparada à população em geral sem diabetes. Evidenciou-se que há relação entre as variáveis amputação e neuropatia. Isso corrobora o estudo de Santos et al. ${ }^{1}$, no que diz respeito às complicações crônicas do diabetes melito, como doença aterosclerótica, alterações na imunidade e presença de neuropatia periférica, que aumentam a incidência e a severidade das complicações da doença, mas esses autores não mencionaram os índices de amputações maiores ou menores. A presença de doença arterial periférica tem sido descrita por alguns autores como fator de risco para amputações em diabéticos ${ }^{1,18}$. Do total de pacientes avaliados, $44,1 \%$ apresentaram alterações de pulsos distais. Quando se relacionou amputações e presença, diminuição e ausência dos pulsos pedioso e tibial posterior, ficou confirmado que quando há alteração desses eventos, o paciente pode evoluir para amputação caso não seja submetido a revascularização. Sendo assim, a doença arterial pode ser considerada como um dos principais fatores de risco para ulceração e, consequentemente, para amputação ${ }^{19}$. A palpação dos pul- sos arteriais distais é considerada um instrumento de grande valia na triagem para doença oclusiva arterial ${ }^{5}$. A ocorrência de úlcera prévia, quando relacionada a amputação, foi considerada fator de risco, já que o odds ratio foi de 14,5, significando que a ocorrência de ulceração anterior aumenta em cerca de 14,5 vezes o risco para amputação. No presente estudo, a classificação de Wagner, escolhida pela simplicidade e facilidade de utilização, foi usada para distribuir as lesões apresentadas pelos pacientes $(96,8 \%$ classificaram como grau $0,2,2 \%$ como grau 1 e $1,1 \%$ como grau 2). Sendo assim, todos podem ser enquadrados como pés em risco. Associou-se essa classificação à ocorrência de amputações, e o odds ratio foi de 44, destacando a importância de tal classificação e sua relação com as amputações de membros inferiores. Segundo Nunes et al. ${ }^{5}$ e Santos et al. ${ }^{1}$, graus 4 e 5, na classificação de Wagner, estão associadas mais frequentemente ao desfecho de amputação. No que se refere à classificação de Texas, não foi possível aplicar nenhum teste estatístico, mas de acordo com a classificação dos pacientes, 95,6\% apresentam risco de ulceração (tipos 0 a 3) e 4,4\% risco de amputação (tipos 4A, 4B, 5 e 6). Sugere-se que, diante dos dados avaliados, seja reforçada a importância do papel do PSF no que se refere à atenção a saúde primária, pois se observou, por meio do índice de amputações, que quando existe assistência adequada à saúde, é possível minimizar a morbimortalidade de doenças como o diabetes melito. Observou-se que a maior parte dos fatores de riscos relacionados é passível de prevenção primária com a provisão de cuidados adequados à saúde ${ }^{4,5,8,20,21}$. Destaca-se o papel do PSF Virgem dos Pobres III, que efetivamente contribuiu para a melhoria da assistência e da qualidade de vida das pessoas, especialmente aquelas com diagnóstico de diabetes melito. Conclui-se que a maneira mais fácil e eficaz ainda é a prevenção. Deve-se ter como objetivo proporcionar atendimento ambulatorial especializado aos diabéticos, com uma equipe multidisciplinar, possibilitando orientação em relação à importância do controle glicêmico, cuidados com os pés e busca de assistência médica tão logo surjam quaisquer lesões em membros inferiores ${ }^{4,5,9,21}$. Para que haja redução do número de amputações, é necessário conhecer detalhadamente as necessidades de saúde dos pacientes e a forma como estão sendo cuidados, para definir o que e quanto precisa ser realizado para melhorar a evolução de tais doentes. 


\section{Conclusão}

Diante dos dados obtidos, destacam-se a prevalência de lesões dermatológicas $(83,9 \%)$ e de neuropatias $(50,5 \%)$, como também de ausência (em 44\%) de pelo menos um dos pulsos na população estudada, a qual poderia receber uma atenção especial para não favorecer a ocorrência de amputações. É de extrema importância a realização de estudos como este para que se possa realizar uma avaliação da ocorrência e dos fatores de risco para amputações, possibilitando o estabelecimento de objetivos para controlar o diabetes melito. Dessa forma, suas principais complicações (como as amputações de extremidades inferiores) seriam reduzidas e diminuiria o impacto previsto da doença, mediante a promoção da saúde, de medicina preventiva e de uma atenção de melhor qualidade, ocasionando consequentemente melhor qualidade de vida para os pacientes.

\section{Referências}

1. Santos VP, Silveira DR, Caffaro RA. Fatores de risco para amputações maiores primárias em pacientes diabéticos. Sao Paulo Med J. 2006;124:66-70.

2. Gamba, MA. Amputações por diabetes mellitus: uma prática prevenível? Acta Paul Enf. 1998;11:92-100.

3. Aguiar, ET. Doença vascular periférica. . 1999;8:971-80.

4. Jorge BH, Borges MF, Brito VN, Santos TG, Thirone AC. Análise clínica e evolução de 70 casos de lesões podais infectadas em pacientes diabéticos. Arq Bras Endocrinol Metab. 1999;43:366-72.

5. Nunes MA, Resende KF, Castro AA, Pitta GB, Figueiredo LF, Miranda Jr F. Fatores predisponentes para amputação de membro inferior em pacientes diabéticos internados com pés ulcerados no estado de Sergipe. J Vasc Bras. 2006;5:123-30.

6. Miyajima S, Shirai A, Yamamoto S, Okada N, Matsushita T. Risk factors for major limb amputations in diabetic foot gangrene patients. Diabetes Res Clin Pract. 2006;71:272-9.

7. Pitta GB, Castro AA, Soares AM, et al. Perfil dos pacientes portadores de pé diabético atendidos no Hospital Escola José Carneiro e na Unidade de Emergência Armando Lages. J Vasc Bras. 2005;4:5-10.

8. de Luccia, N. Doença vascular e diabetes. J Vasc Bras. 2003;2:49-60.

9. Gamba MA, Gotlieb SLD, Bergamaschi DP, Vianna LAC. Amputações de extremidades inferiores por diabetes melli- tus: estudo caso-controle. Rev Saude Publica. 2004;38:399404.

10. Spichler D, Miranda Jr F, Spichler ES, Franco LJ. Amputações maiores de membros inferiores por doença arterial periférica e diabetes melito no município do Rio de Janeiro. J Vasc Bras. 2004;3:111-22.

11. Consenso Brasileiro sobre Detecção e Tratamento das Complicações Crônicas de Diabetes Melito. Arq Bras Endocrinol Metab. 1999;43:7-20.

12. Sociedade Brasileira de Cardiologia, Sociedade Brasileira de Hipertensão, Sociedade Brasileira de Nefrologia. V Diretrizes Brasileiras de Hipertensão Arterial. http://www.sbn.org.br/Diretrizes/V_Diretrizes_Brasileiras_ de_Hipertensao_Arterial.pdf. Acesso: 03/04/2008.

13. Brasileiro JL, Oliveira WT, Monteiro LB, et al. Pé diabético: aspectos clínicos. J Vasc Bras. 2005;4:11-21.

14. Apelqvist J, Larsson J. What is the most effective way to reduce incidence of amputation in the diabetic foot? Diabetes Metab Res Rev. 2000;16 Suppl 1:S75-83.

15. Moss SE, Klein R, Klein BE. The prevalence and incidence of lower extremity amputation in diabetic population. Arch Intern Med. 1992;152:610-6.

16. Malerbi DA, Franco LJ. Multicenter study of the prevalence of diabetes mellitus and impaired glucose tolerance in the urban Brazilian population aged 30-69 yr. The Brazilian Cooperative Group on the Study of Diabetes Prevalence. Diabetes Care. 1992;15:1509-16.

17. Ministério da Saúde. Consenso brasileiro sobre diabetes diagnóstico e classificação do diabetes mellitus e tratamento do diabetes mellitus tipo 2: recomendações da Sociedade Brasileira de Diabetes. Brasília: Ministério da Saúde; 2000.

18. Carvalho FS, Kunz VC, Depieri TZ, Cervelini R. Prevalência de amputação em membros inferiores de causa vascular: análise de prontuários. Arq Cienc Saude Unipar. 2005;9:23-30.

19. Margolis DJ, Allen-Taylor L, Hoffstad O, Berlin JA. Diabetic neuropathic foot ulcers and amputation. Wound Repair Regen. 2005;13:230-6.

20. Jeffcoate WJ. The incidence of amputation in diabetes. Acta Chir Belg. 2005;105:140-4.

21. Coelho JC. Prevenção de amputação de membros inferiores no paciente diabético. J Bras Med. 2004;87:11-6.

Correspondência:

Elvira Cancio Assumpção

Condomínio Aldebaran Beta, Qd. S, 07, Tabuleiro dos

Martins

CEP 57080-900 - Maceió, AL

Tel.: (82) 3358.5434, (82) 9309.2696

E-mail: elviracancio@hotmail.com 